\title{
Exploring How Trainers Can Use Humor to Increase Self-Efficacy of Employees
}

\author{
Christian A. Homan \\ Wayland Baptist University \\ Angelica R. Landry \\ Wayland Baptist University \\ Sheron Lawson \\ Wayland Baptist University
}

Organizational success depends upon the effective execution of skills and usage of resources. It is the responsibility of workplace leadership to respond effectively to training needs, insufficiencies, and changes that affect the learning culture. One of the purposes of this research is to identify how these humor qualities might influence training and learning efforts.

In addition to conventional approaches, making use of certain humor traits and styles benefit learning cultures since certain humor styles foster and cultivate self-efficacy. Employees with high self-efficacy gain more from training and contribute to the overall effectiveness of the organization by creating better products.

\section{INTRODUCTION}

The workforce consists of employees with a multitude of abilities, skillsets, which requires trainers to incorporate a variety of training methods and motivational techniques. In addition to conventional approaches, using certain humor traits and styles could benefit employee learning and retention of training content since certain humor styles foster and cultivate self-efficacy (Yip \& Martin, 2006). Training is only as useful as what employees take away and use in roles throughout the organization. While self-efficacy describes individuals' confidence that they can do something, instrumentality refers to whether they feel it is worth it and if something will result in what individuals want. Those with high selfefficacy tend to have higher expectations for themselves and are thus more motivated to learn and use new skills thus improving performance (Chiaburu \& Lindsay, 2008; Sun et al., 2016). Both self-efficacy, the can do and instrumentality, will do; aid in learning and motivation of trainees. Smith \& Berge (2009) suggested that trainers who incorporate humor maybe able to enhance the self-efficacy of trainees.

Past research shows that affiliative humor and self-enhancing are positively correlated to selfefficacy. Affiliative humor causes no harm, is used to enhance relationships with others, and supports 
positive thinking (Zeigler-Hill et al., 2016). This point encourages others to get involved in creative problem solving as they feel encouraged by their environment and peers. People who use affiliative humor often say amusing things, tell unthreatening jokes, and generally lighten the mood. Such individuals give humor a bonding purpose by laughing at their own faults (Zeigler-Hill et al., 2016). Humor is thought to be a significant part of individual strength and performance; workers characterized as humorous maintained more significant effort and persisted at their assigned task, making almost double the number of attempts and spending at least fifty percent longer on average than those in other conditions (Wanzer et al., 2005).

Employees' expectations and attitudes also influence the level of effectiveness resulting from any training (Tannenbaum et al., 1991). Part of a firm's strategy to improve learning includes those training the workforce. As professionals and institutions integrate with and depend more on technology, new concepts of social learning are being formed through distance learning and computer-based training. Despite this, there is still a social aspect that is unavoidable and may be enhanced through the use of humor.

\section{Problem Statement}

Encouraging self-efficacy in organizations may benefit retention and organizational performance. Researching the four styles of humor may offer insights into how they can influence training, and, by extension, self-efficacy. Although most corporations are making better use of advanced programs in their training schedules, employees' low self-efficacy and self-worth can stymie the training received. If employers do not excel at providing their employees necessary tools, appropriate feedback, and employable skills, then the organization's performance standards will fail the workforce. Perhaps trainers who incorporate humor can enhance the self-efficacy of trainees (Smith \& Berge, 2009).

\section{Purpose}

This research project hypothesizes that if trainers incorporate humor as training tool, it can enhance the self-efficacy of the trainees. Enhanced self-efficacy can help in the acceptance and retention of training content which correlate with reduced voluntary employee turnover.

The major objective of this paper is to explore the four humor models and propose how their characteristics can influence self-efficacy in the training process to lead to enhanced learning and retention of organizational knowledge. By focusing on humor facets in the workplace, training systems and organizational may be optimized more often (Falanga et al., 2014).

\section{Propositions}

Proposition 1: If trainers incorporate affiliative and self-enhancing humor, they will positively influence self-efficacy.

Proposition 2: Increased self-efficacy and self-enhancing can increase employee motivation leading to transfer of training to organizational processes.

\section{LITERATURE REVIEW}

\section{The Relevance of Humor in Organizations}

Humor is thought to be a significant part of individual strength and performance; workers characterized as humorous maintained more significant effort and persisted at their assigned task, making almost double the number of attempts and spending at least fifty percent longer on average than those in other conditions (Wanzer et al., 2005). However, this attribute may vary in accordance with types of humor and job environments. Scholars generally categorize humor into four distinct styles, two that embody a positive energy (aggressive and affiliative), and two that embody a negative energy (selfdefeating and self-enhancing). Aggressive humor is used to enhance the self and cause harm because it 
focuses on the perceived superiority of the user over others; this style attempts to inflate the self at the expense of others. Another description of aggressive humor is put-down humor, which makes others look bad so the speaker looks and feels better (Zeigler-Hill, V., McGabe, G. A., \& Vrabel, J. K., 2016).

Past research shows that affiliative humor causes no harm, is used to enhance relationships with others, and supports positive thinking (Zeigler-Hill et al., 2016). This point encourages others to get involved in creative problem solving as they feel encouraged by their environment and peers. People who use affiliative humor often say amusing things, tell unthreatening jokes, and generally lighten the mood. Such individuals give humor a bonding purpose by laughing at their own faults (Zeigler-Hill et al., 2016).

Self-defeating humor is considered damaging because it is used to enhance relationships with others and receive attention by making fun of oneself in disparaging or undignified ways. This style of humor is employed by those who want to make themselves prominent, even if it means through negative associations. Self-defeating humorists make themselves the butt of the joke for the amusement of others (Zeigler-Hill et al., 2016).

The fourth expression of humor is called self-enhancing humor and is used to enhance the self. This style is used as an upbeat coping strategy to effectively deal with stress by uplifting self-confidence and, as a frequent result, creativity. Self-enhanced humorists often do not take themselves too seriously, help to cope with challenges by taking a broad look at the situation, and laugh at the weirdness of the cosmos and everyday life (Amjed \& Tirmzi, 2016).

Humor is a tool that helps in enhancing employee potential, so knowing when and how to best use specific forms of humor in the workplace may help those with difficulty communicating humor (Wanzer et al., 2005). Positive and negative styles of self-directed humor are assumed to have opposing relationships with perceived stress, but the connections are predicted to be mediated through positive personality qualities.

One approach, called superiority theory, describes humor as a method to achieve superiority and secure control by laughing at people or objects via mocking, making ethnic comments and jokes, and putting down others. Another approach, incongruity theory, suggests that humor is caused by surprises and exceptional circumstances (inconsistencies), such as when differences between bodies of disparate knowledge are resolved. One more approach, called relief theory, observes that humor causes laughter, which benefits by releasing tension (Romero \& Arendt, 2011).

\section{The Role of Self-efficacy}

Low self-efficacy prevents employees from benefiting from organizational training and can damage organizational performance and team productivity. Researchers describe self-efficacy in the literature as individuals' belief that they can accomplish a behavior or task (Chiaburu \& Lindsay, 2008; Dar, EbelLam, \& Douchet, 2018; Lloyd, Bond, \& Flaxman, 2016; Noe, 1986; Sun, Chen, \& Song, 2016; Tannenbaum, Mathieu, Salas, \& Cannon-Bowers, 1991; Yoon \& Kayes, 2016). This is valuable to an organization because those who believe they can do something are more likely to do it and continue completing tasks. By actively working on new and familiar tasks, the workforce will likely improve as the adage "practice makes perfect" suggests.

Navigating a team through the training process involves, among other stages, painting a compelling picture, effectively communicating, getting rid of obstacles, empowering groups and individuals, and making changes stick (Daft, 2015). Factors that exacerbate or mitigate the challenges of training often lead to pressure, high workload, ineffective management, secrecy, blame culture, uncomfortable or unpleasant physical working environment, job uncertainty, unhealthy work relationships, lack of career development, weak leadership, and insufficient free time (Rich, 2016).

Low self-efficacy encountered in the work environment is linked with decreased work performance, reduced interpersonal skills, lack of focus, poor teamwork, and decreased productivity (Rich, 2016). Organizational leaders stand to benefit by empowering their human resource personnel to be creative and depend on their initiative and creativity. Forward thinking in this context leads to venturing beyond traditional hiring methods to find talent, making the most of social networking, and knowing what kind of 
qualities and work relationships are needed by the organization. Few changes strike fear into the hearts of employees quite like losing a job they expect to keep.

The way employees express themselves and communicate via humor affects the entire organization. Humorous people access more categories of communication behaviors such as nonverbal techniques, language, expressivity, and impersonation. Those who enacted humor more frequently utilized a variety of communication behaviors in a wider range of situations than those who did not (Wanzer et al., 2005).

\section{How Humor Influences Self-efficacy}

A leader's perception stands at the heart of organizational improvement. An effective leader is ready to gain self-awareness from a range of sources. Awareness might be developed through dynamic interaction with others, being open to the signals others give, and sensitivity to humor cues. Selfawareness helps professionals understand who they are and what they want to achieve. Rather than becoming defensive about their fears and inadequacies, they decide to transform themselves and thrive (Showry \& Manasa, 2014).

Meyer (2000) elucidated that humor has a unifying effect through identification which can bring a speaker symbolically closer to an audience. Identification and clarification functions of humor provide reassurance while also providing humorous divergence from normality of situation. By showing that group members can laugh at situations made them seem relaxed and more in control, potentially boosting group confidence. Meyer (2000) further stated that this leads to feelings of unity as "in-group-ness," through the sharing of a particular joke or story

An individual's self-awareness may correlate with how they employ humor to suit their needs and desires. Individuals with high levels of negative affectivity refrain from using affiliative and selfenhancing forms of humor because of how these attempts at humor will be interpreted by others, while individuals with high levels of detachment usually lack the motivation to enhance themselves or their relationships with others (Zeigler-Hill et al., 2016).

Scheel and Gockel, (2017) described the social functions of humor as lubricating and that laughter, may create closeness between individuals. Beyond providing amusement, humor can facilitate liking and can bring people together. Scheel and Gockel, (2017) explicated that management humor has influence on group characteristics such as cohesiveness, communications, power, and status. The interpersonal consequences of humor at work encompass attention, immediacy, cohesion, emotional contagion, status and face-saving. For instance, relying on the face-saving ambiguity of humor may enable groups to resolve conflict without engaging in destructive behavior. Humor, cited Scheel and Gockel, (2017), is very beneficial in the work and in training and can promote health, enhance mental flexibility, and smooth social relations.

\section{CONCLUSION}

A goal of this project was to examine how different forms of humor influence employees' learning and retention of training content. Humor is not only an avenue for individuals to explore entertainment, share jokes, and harmlessly deviate from social norms, but forms of humor may even indicate spiritual generosity, mental health, and creativity (Falanga et al., 2014). The finding from the reviewed of literature correlate affiliative and self-enhancing humor to self-efficacy by identifying how the many stresses that face organizations from growing diversity, miscommunication, and misaligned goals between colleagues can be mitigated by creating or maintaining group cohesiveness.

Affiliative humor which enhances relationships with others and supports positive thinking was found to influence teamwork and creative problem solving (Zeigler-Hill et al., 2016). Self-enhancing humor which helps in coping strategy and uplifting self-confidence, was also found to aid in motivation and creativity (Amjed \& Tirmzi, 2016).

Humor was found to be a unifying effect and is instrumental in stimulating group characteristics such as cohesiveness, communications, power, and status which enable groups to resolve conflict without engaging in destructive behavior (Meyer, 2000; Scheel and Gockel, 2017). Understanding the way humor 
styles resonate throughout organizations and affect individuals may help to control employee turnover. Decision-makers and training managers may, through studies of this type, steer their culture in ways to attract specific humor types. Scheel and Gockel, (2017) stated that humor is very beneficial in the work and in training and can promote health, enhance mental flexibility, and smooth social relations.

Individuals engaging in activities that require persistence may benefit from possessing certain humorous traits. Organizations that acknowledge the value of self-enhancing humor might create stronger, more effective organizations through employees' increased persistence. Assembling the right talent that stands to withstand changes, challenges, and uncertainties in the workplace remain one of the top priorities of employers.

The research findings corroborate the first proposition that if affiliative and self-enhancing humors are incorporating in training, they will positively influence self-efficacy. The results also confirmed the second proposition that increased self-efficacy and self-enhancing can increase employee motivation leading to transfer of training to organizational processes.

\section{REFERENCES}

Amabile, T. M., \& Conti, R. (1999). Changes in the work environment for creativity during downsizing. Academy of Management Journal, 42(6), 630-640.

Amjed, A., \& Tirmzi, S. H. S. (2016). Effect of humor on employee creativity with moderating role of transformational leadership behavior. Journal of Economics, Business and Management, 23(1), 41-50.

Baker, S. D., Mathis, C. J., Stites-Doe, S., \& Javadian, G. (2016). The role of trust and communication in fostering followers' self perceptions as leaders. Journal of Managerial Issues, 28(3-4), 210-222.

Behfar, K. J., Peterson, R. S., Mannix, E. A., \& Trochim, W. M. K. (2008). The critical role of conflict resolution in teams: A close look at the links between conflict type, conflict management strategies, and team outcomes. Journal of Applied Psychology, 93(1), 170-188.

Chiaburu, D. S., \& Lindsay, D. R. (2008). Can do or will do? The importance of self-efficacy and instrumentality for training transfer. Human Resource Development International, 11(2), 199206. doi:10.1080/13678860801933004

Daft, R. L. (2015). The leadership experience. Cengage Learning.

Darr, W. A., Ebel-Lam, A., \& Doucet, R. G. (2018). Investigating the extravert advantage in training: Exploring reward sensitivity, training motivation, and self-efficacy as intermediary factors. Canadian Journal of Behavioural Science / Revue Canadienne Des Sciences Du Comportement, 50(3), 172-184. doi:10.1037/cbs0000102

Falanga, R., De Caroli, M. E., \& Sagone, E. (2014). Humor styles, self-efficacy and prosocial tendencies in middle adolescents. Procedia - Social and Behavioral Sciences, 127, 214-218.

Grosser, T. J., Venkataramani, V., \& Labianca, G. (2017). An alter-centric perspective on employee innovation: The importance of alters' creative self-efficacy and network structure. Journal of Applied Psychology, 102(9), 1360-1374. doi:10.1037/ap10000220

Harryson, S. J. (2008). Entrepreneurship through relationships - navigating from creativity to commercialization. $R \& D$ Management, 38(3), 290-310.

Ismail, A., Mohd Zainol, N. A., \& Ahmad, N. N. (2017). The administration of leadership training programs enhance the trainee's motivation to learn. Logforum, 13(4), 465-477. doi:10.17270/J.LOG.2017.4.7

Lloyd, J., Bond, F. W., \& Flaxman, P. E. (2017). Work-related self-efficacy as a moderator of the impact of a worksite stress management training intervention: Intrinsic work motivation as a higher order condition of effect. Journal of Occupational Health Psychology, 22(1), 115-127. doi:10.1037/ocp0000026

Nezlek, J. B., \& Derks, P. (2001). Use of humor as a coping mechanism, psychological adjustment, and social interaction. Humor, 14(4), 395-413.

Journal of Organizational Psychology Vol. 19(3) 2019125 
Noe, R. A. (1986). Trainees' attributes and attitudes: Neglected influences on training effectiveness. The Academy of Management Review, 11(4), 736-749.

Rich, S. (2016). A brief examination of the effects of occupational stress on creativity and Innovation. The Psychologist-Manager Journal, 19(2), 107-121.

Romero, E. J., \& Arendt, L. A. (2011). Variable effects of humor styles on organizational outcomes. Psychological Reports, 108(2), 649-659.

Showry, M., \& Manasa, K. V. L. (2014). Self-awareness - Key to effective leadership. IUP Journal of Soft Skills, 8(1), 15-26.

Smith, M., \& Berge, Z. L. (2009). Social learning theory in second life. MERLOT Journal of Online Learning and Teaching.

Sun, S., Chen, H., \& Song, Z. (2016). Cross-level moderating effects of conscientiousness on within person relationships of self-efficacy to effort allocation. Human Performance, 29(5), 447-459.

Tannenbaum, S. I., Mathieu, J. E., Salas, E., \& Cannon-Bowers, J. A. (1991). Meeting trainees' expectations: The influence of training fulfillment on the development of commitment, selfefficacy, and motivation. Journal of Applied Psychology, 76(6), 759-769.

Yip, J. A., \& Martin. A. (2006). Sense of humor, emotional intelligence, and social competence. Journal of Research in Personality, 40(6), 1202-1208.

Yoon, J., \& Kayes, D. C. (2016). Employees' self-efficacy and perception of individual learning in teams: The cross-level moderating role of team-learning behavior. Journal of Organizational Behavior, 37(7), 1044-1060. doi:10.1002/job.2092

Zeigler-Hill, V., McCabe, G. A., \& Vrabel, J. K. (2016). The dark side of humor: DSM-5 pathological personality traits and humor styles. Europe's Journal of Psychology, 12(3), 363-376.

Wanzer, M., Booth-Butterfield, M., \& Booth-Butterfield, S. (2005). "If we didn't use humor, we'd cry": humorous coping communication in health care settings. Journal of Health Communication, $10(2), 105-125$. 